\title{
The Role of the Lateral Intraparietal Area of the Monkey in the Generation of Saccades and Visuospatial Attention
}

\author{
MICHAEL E. GOLDBERG,${ }^{a, c}$ JAMES BISLEY,${ }^{a, c}$ KEITH D. POWELL ${ }^{a}$ \\ JACQUELINE GOTTLIEB, ${ }^{a}$ AND MAKOTO KUSUNOKI ${ }^{a, b}$ \\ ${ }^{a}$ The Laboratory of Sensorimotor Research, National Eye Institute, \\ Bethesda, Maryland 20892-4435, USA \\ ${ }^{b}$ University College, London, United Kingdom \\ ${ }^{c}$ David Mahoney Center for Mind and Brain, Center for Neurobiology and Behavior, \\ Departments of Neurology and Psychiatry, Columbia University, and the \\ New York State Psychiatric Institute, New York, New York, USA
}

\begin{abstract}
The brain cannot monitor or react towards the entire world at a given time. Instead, using the process of attention, it selects objects in the world for further analysis. Neuronal activity in the monkey intraparietal area has the properties appropriate for a neuronal substrate of attention: instead of all objects being represented in the parietal cortex, only salient objects are. Such objects can be salient because of their physical properties (recently flashed objects or moving objects) or because they can be made important to the animal by virtue of a task. Although lateral intraparietal area (LIP) neurons respond through the delay period of a memory-guided saccade, they also respond in an enhanced manner to distractors flashed during the delay period of a memoryguided saccade being generated to a position outside the receptive field. This activity parallels the monkey's psychophysical attentional process: attention is ordinarily pinned at the goal of a memory-guided saccade, but it shifts briefly to the locus of a task-irrelevant distractor flashed briefly during the delay period and then returns to the goal. Although neurons in LIP have been implicated as being directly involved in the generation of saccadic eye movements, their activity does not predict where, when, or if a saccade will occur. The ensemble of activity in LIP, however, does accurately describe the locus of attention.
\end{abstract}

KEYWORDS: parietal; saccade; monkey; attention; salience

\section{INTRODUCTION}

We live in a world of sensory overload. Sights, sounds, smells, and touches bombard our sensory apparatus constantly, and the primate brain cannot possibly deal with all of them simultaneously. Instead, it chooses among this intense sensory

Address for correspondence: Michael E. Goldberg, The Laboratory of Sensorimotor Research, National Eye Institute, 49 Convent Drive, Room 2A-50, Bethesda, MD 20892-4435, USA. Voice: 301-496-1060; fax: 301-402-0511.

meg@1sr.nei.nih.gov

Ann. N.Y. Acad. Sci. 956: 205-215 (2002). () 2002 New York Academy of Sciences. 
world the objects most relevant to its behavior for further processing. This act of selection is called attention. James ${ }^{1}$ described attention as "the taking possession by the mind in clear and vivid form, of one out of what seem several simultaneously possible objects or trains of thought.... It implies withdrawal from some things in order to deal effectively with others." He then described two different kinds of attention: "It is either passive, reflex, non-voluntary, effortless or active and voluntary. In passive immediate sensorial attention the stimulus is a sense-impression, either very intense, voluminous, or sudden...big things, bright things, moving things...blood."

More recently, these two kinds of attention have been described as exogenous and endogenous. Primates usually look at the objects of visual attention, and investigators have described two different kinds of attention along a different axis, depending on whether the subject actually looks at the object (covert attention) or responds without looking (overt attention). ${ }^{2}$

The parietal cortex has long been thought to be important in the neural mechanisms underlying spatial attention. One area in particular, the lateral intraparietal area (LIP), has been implicated in attentional and oculomotor processes. Although it is clear that LIP has a visual representation, it is not clear if this visual representation is dedicated to processing saccadic eye movements or if it has a more general attentional function independent of the generation of any specific movement. In this review, we describe three different experiments that examine the role of attention in LIP and its relation to the generation of saccadic eye movements. The first deals with the nature of the visual representation in LIP, the second with the independence of LIP activity from saccade planning, and the third with the nature and determinants of visual attention in the monkey. We begin with a description of the methods common to all three experiments.

\section{GENERAL METHODS}

Six rhesus monkeys were trained to perform various visual tasks for liquid reward and then were prepared, under sterile surgical procedures, for neurophysiological and eye position recording. All animal protocols were approved as conforming to the National Institutes of Health guidelines for animal care and use by the National Eye Institute Animal Care and Use Committee. We recently described our physiological, anatomical, and data analytical methods elsewhere. ${ }^{3,4}$

The monkeys were trained to fixate on a red laser spot that appeared on a tangent screen $86 \mathrm{~cm}$ in front of them. They were rewarded for maintaining their eye within a fixation window ( 2 degrees) in width. When the fixation point moved, the monkeys followed it with a saccade. ${ }^{5}$ They also quickly learned a memory-guided delayed saccade task: while the monkey looked at the central fixation point, a peripheral stimulus was flashed for $200 \mathrm{~ms}$. After a delay of 500-1,000 ms, the fixation point disappeared and the monkey made saccades to the remembered spatial location of the now vanished target. ${ }^{6}$ Having learned these standard tasks, the monkeys were ready to learn tasks that were more complicated, that is, the stable array, the distractor, and the go-no-go saccade tasks. 


\section{NATURE OF VISUAL REPRESENTATION IN THE LATERAL INTRAPARIETAL AREA}

Since the development of the fixation task by Wurtz, ${ }^{7}$ the standard method for determining a visual response of a neuron has been the response of the neuron to a stimulus that appears suddenly in its receptive field. This definition has a problem, however. Abruptly appearing stimuli are not only associated with photons exciting rods and cones; as James ${ }^{8}$ noted, they are also attentional attractors. Stimuli can enter receptive fields in several ways: one is when a light appears suddenly in the receptive field; a second is when a saccade brings a stable object into the receptive field. Since activity in the parietal cortex is associated with attention as well as with vision, the question arises as to whether the 'visual responses' of parietal neurons are visual, that is, responding to photons on the retina, like a retinal ganglion cell, or attentional.

To distinguish between these alternatives we devised a number of tasks in which the stimulus, rather than appearing de novo in the receptive field, entered the receptive field by virtue of a saccadic eye movement. This enabled us to stimulate the receptive field using stimuli that did not have the attentional tag of abrupt onset. In these stable array tasks, ${ }^{9}$ the monkeys were presented with an array of eight stimuli arranged uniformly in a circular array. These stimuli did not appear or disappear from trial to trial. Instead, they were constant for a block of trials. The stimuli were roughly 2 degrees in diameter and varied in shape and color. They were not equated for luminance. They were positioned so that when the monkey fixated on the center of the array, at least one stimulus appeared in the receptive field of the neuron under study. In the simplest of these tasks the monkey fixated at a position outside the array so that no stimulus was in the receptive field of the neuron being studied, and then, when the red fixation point jumped, the monkey made a saccade to the center of the array (FIG. 1). This saccade brought one of the stable stimuli into the receptive field.

The typical neuron had a brisk response to the sudden appearance of a stimulus in its receptive field during a fixation task (FIG. 2A), and a much smaller response when the same stimulus as a member of the stable array entered the receptive field (FIG. 2B). The decrement of response could have been related to the behavioral irrelevance of the stable target or it could have been due to a series of other confounds. For example, the movement of the stimulus into the receptive field by the saccade is not exactly the same as its appearance from the flash; the other members of the array might exert some purely visual local inhibition that suppresses the response. To test if these other factors could be responsible for the diminished response to the stable target, we developed the recently flashed stimulus task. In this task, the stable array contained only seven stimuli, but not the one that would be brought into the receptive field by the saccade. This eighth stimulus appeared while the monkey was fixating at the initial position, and it remained on throughout the trial. The monkey then made a saccade that brought this recently appeared stimulus into the receptive field. The neuron responded almost as briskly in that case as it did to the abrupt appearance of the stimulus in the receptive field (FIG. 2C; compare with FIG. 2A). Therefore, the difference between the fixation case and the stable target case was due not to the visual or oculomotor differences between the tasks, but to the lack of salience of a stable component of the visual environment. Note that the neuron began to respond at or before the end of the saccade. This was a much lower latency than that when the 


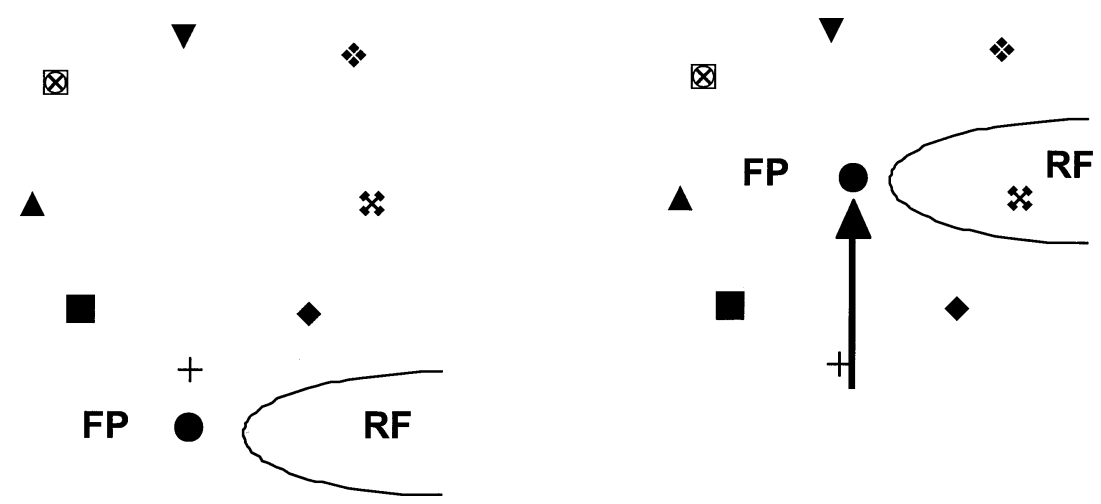

FIGURE 1. Stable array task. An array of symbols remains on the screen unchanging throughout the task. (Left) The monkey looks at a fixation point (black dot, marked FP) situated so no member of the array is in the receptive field (parabolic solid line, RF) of the neuron. (Right) The fixation point jumps and monkey makes a saccade (arrow) to follow it, bringing the receptive field onto the spatial location of a symbol (in this case the X). Adapted from Gottlieb et al. ${ }^{9}$

A

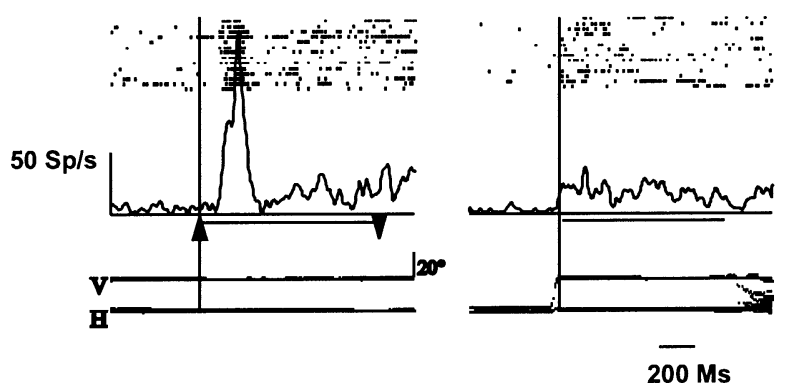

C
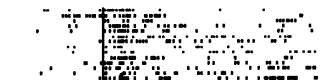

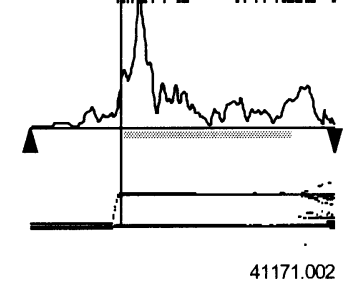

FIGURE 2. Effect of recent flash on stable array response. Each diagram is a raster diagram. Each dot is a cell discharge. Each line represents cell activity for one trial. Successive lines are synchronized on an event that occurs at the vertical line. Spike density histograms are shown beneath each raster. The gray bar at the bottom of the spike density histogram shows when, during the trial, the stimulus is in the receptive field of the neurons. Up arrows represent the onset of the flashed stimulus; down arrows represent its disappearance. Horizontal $(\mathrm{H})$ and vertical $(\mathrm{V})$ eye position traces for each raster line are shown superimposed beneath the spike density diagram. (A) Stimulus flashes in receptive field during fixation task; activity synchronized on stimulus appearance. (B) Stable array task: monkey makes saccade that brings stable stimulus into receptive field; activity synchronized on saccade end. (C) Recent stimulus task: monkey makes saccade that brings recently flashed stimulus into receptive field. Stimulus appears at up arrow, roughly $500 \mathrm{~ms}$ before saccade; activity synchronized on saccade end. Adapted from Gottlieb et al. ${ }^{9}$ 


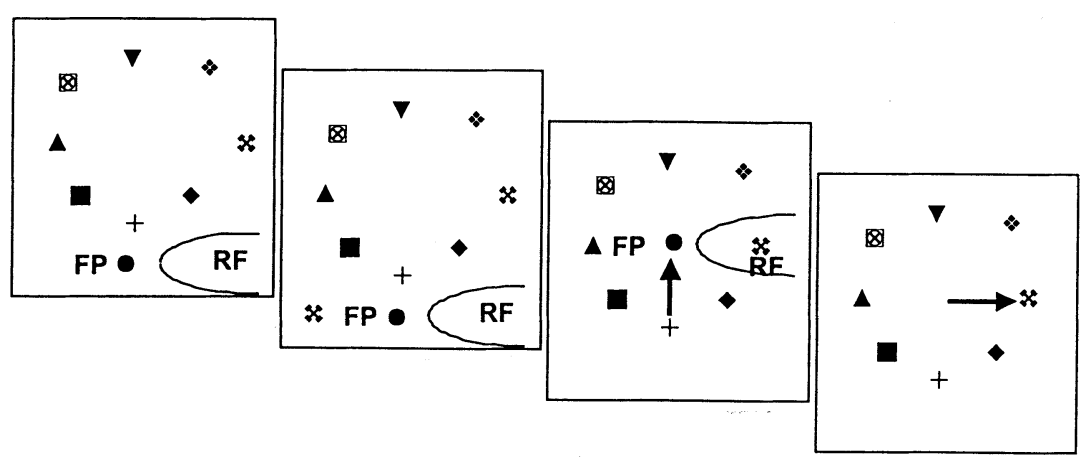

FIGURE 3. Stable target task. (First panel) The monkey fixates so that all symbols in the array are outside the receptive field. (Second panel) A cue appears, also outside the receptive field. (Third panel) The fixation point jumps, and the monkey makes a saccade that brings a symbol into the receptive field. In this example the symbol in the receptive field matches the cue. (Fourth panel) The fixation point disappears, and the monkey makes a saccade to the symbol that matches the cue. Adapted from Gottlieb et al. ${ }^{9}$

stimulus appeared in the receptive field abruptly (compare FIG. 2A with FIG. 2C). Presumably this occurred because of the predictive response described previously ${ }^{10}$ : neurons in LIP may respond to stimuli that will be brought into their receptive field by saccades earlier than they respond to the abrupt appearance of the same stimulus in their receptive fields. The recently appeared stimulus evoked a greater response across the population than did the stable stimulus (using an average response in an interval $200 \mathrm{~ms}$ after the end of the saccade: $p<0.001$ by Wilcoxon signed rank test; 31 neurons), and it evoked a statistically significantly greater response in a majority $(23 / 31, p<0.05$ by two-tailed $t$ test) of single neurons.

Salience does not only arise from intrinsic properties of the stimulus. Stable objects can become important by virtue of their relevance to current behavior, and under those circumstances a member of a stable array can evoke a response from a neuron in LIP. We can show this using the stable target task, a more complicated version of the stable array task (FIG. 3). In this task, the monkey fixated so that the stimulus was not in the receptive field, and a cue appeared during the first fixation. This cue matched one of the symbols in the stable array. The fixation point then jumped to the center of the array and the monkey tracked it with a saccade. Finally, when the fixation point disappeared, the monkey made a saccade to the member of the array that had matched the cue. The target indicated by the cue was randomly chosen on each trial among the members of the array. Neurons responded strongly to stable stimuli brought into their receptive field if these were designated as the target of the next saccade (FIG. 4A). The neuron discharged from the first saccade to the second. By contrast, if the identical stable stimulus entered the receptive field but was not designated as the saccade target (the monkey was instructed to saccade elsewhere), neurons responded minimally (FIG. 4B).

It is possible that LIP neurons respond in the stable target task because the monkey is planning a purposive saccade, ${ }^{11}$ and the activity is less related to the salience of the stable target than it is to the processes underlying saccade planning. To see 
A

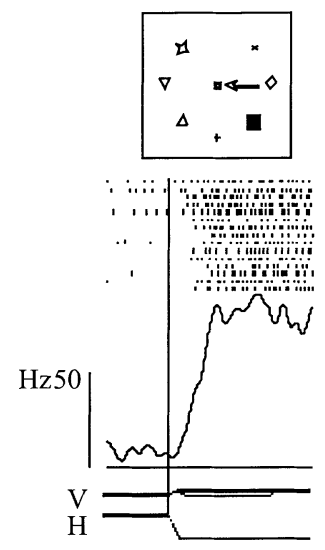

First Saccade
B

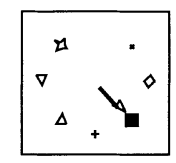

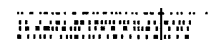

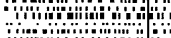

(1)
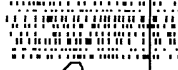

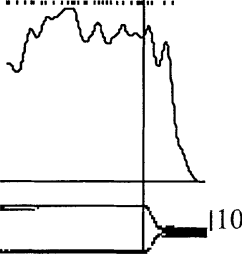

Second Saccade
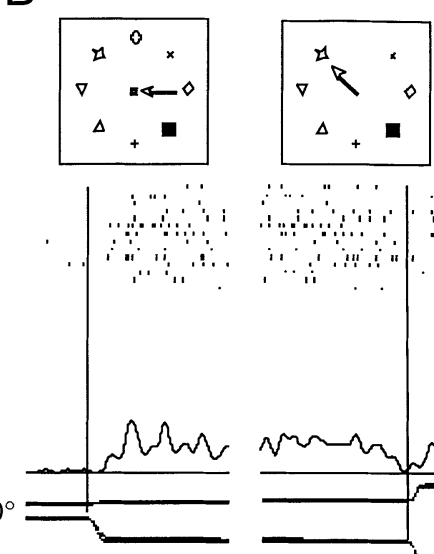

$\therefore \cdots \cdots$

a

i i.
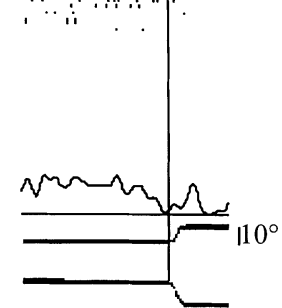

First

Second Saccade

FIGURE 4. Response of the neuron in the stable target task. Each pair of rasters shows the response of the neuron in the same trials synchronized on first saccade beginning (left) and second saccade beginning (right). (A) Second saccade to receptive field. Cue appears before the first saccade and matches stable symbol in the receptive field. (B) Second saccade to receptive field. Cue matches target 180 degrees away from receptive field. Adapted from Kusunoki et al. ${ }^{3}$

how much activity in LIP can be allocated to the planning and generation of a saccade itself we used a task in which the monkey had to make a saccade to a spatial location that had no visual stimulus (the "black hole" task). LIP neurons active in the stable target task frequently failed to respond in this task, and no neurons responded more in this task than in the stable target task when the cue, and not the target, was in the receptive field. ${ }^{9}$

\section{EFFECT OF SACCADE PLANNING ON VISUAL RESPONSES OF NEURONS IN THE LATERAL INTRAPARIETAL AREA}

It could be, however, that LIP is only dedicated to the planning of visually guided saccades, and learned saccades such as those in the black hole task are irrelevant to LIP. If LIP were exclusively involved in planning saccades, then once the monkey was committed to a given saccade, a stimulus appearing far away from the saccade goal should evoke a weaker response than a stimulus at the saccade goal. ${ }^{4}$ To test this we first studied neurons in the delayed saccade task and only chose those that had delay period and/or presaccadic activity (FIG. 5A and B). The neuron illustrated in FIGURE 5 has a visual response, a striking delay period response but a lesser presaccadic increase. 


\section{A. Delayed Saccade Stimulus Onset}

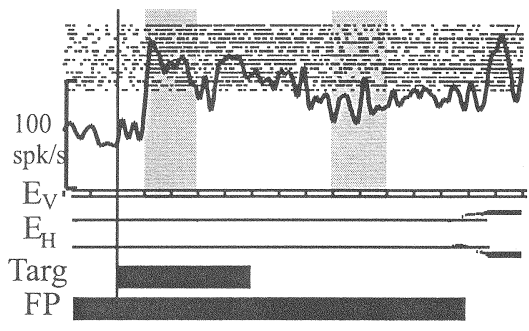

\section{Saccade out of RF Distractor in RF}

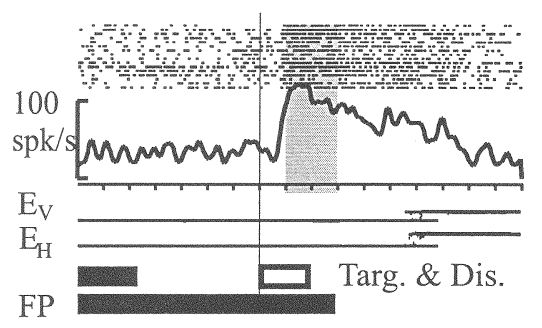

\section{B. Delayed Saccade Saccade Onset}

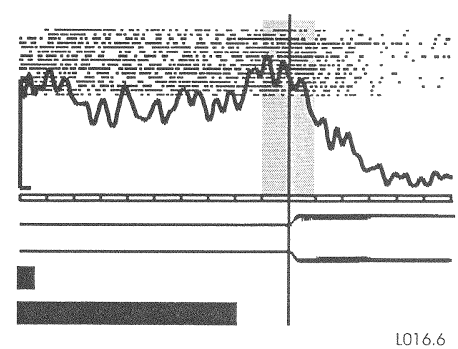

\section{Saccade to RF Distractor in RF}

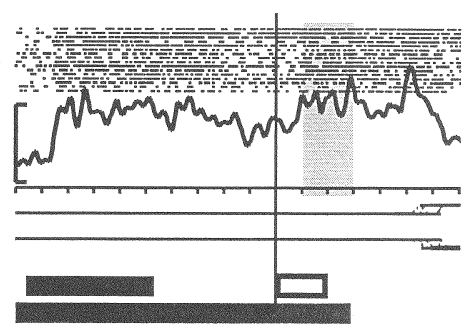

L016.6

FIGURE 5. Response of a neuron to a distractor flashed in its receptive field. Each panel shows a raster, spike density histogram, and stimulus traces for fixation point, saccade target, and distractor. Shaded areas in the raster show stimulus, delay, and distractor activity periods used for quantitative analyses. (A) Response in the delayed saccade task synchronized on the stimulus appearance. (B) Same activity synchronized on the saccade beginning. (C) Response of the same neuron to the stimulus flashed in the receptive field (RF) during the delay period of a saccade made outside the receptive field. (D) Response of the same neuron during the delay period of a saccade made to the receptive field. Adapted from Powell and Goldberg. ${ }^{4}$

We then studied 27 neurons with visual and delay and/or presaccadic activity in the distractor task. In this task the monkey performs a delayed saccade task, but 300 $\mathrm{ms}$ before the fixation disappeared we flashed a distractor for $200 \mathrm{~ms}$ that was identical to the saccade target. We randomly interleaved six types of trials: simple delayed saccade tasks to the receptive field or to a second point that was outside the receptive field; trials with saccades to the receptive field and distractor either in the receptive field or away; and trials with saccades away from the receptive field and the distractor either in the receptive field or at the saccade goal.

We found that when a stimulus flashed in the receptive field of an LIP neuron while the monkey was planning a memory-guided saccade away from the receptive 
field, not only was the response of the neuron not suppressed, but also it was slightly enhanced relative to the case when the stimulus appeared at the saccade goal. FIGURE $5 \mathrm{C}$ and D illustrates this for the same neuron whose activity in the memory-guided saccade task was shown in Figure 5A and B. This was true across the population of neurons and for individual neurons as well. If one looked at the activity of the neurons in the $50 \mathrm{~ms}$ before the beginning of the saccade, no difference was noted between the case when the monkey made a saccade to the receptive field and the distractor was elsewhere and the case when the monkey made a saccade away from the receptive field and the distractor was in the receptive field. Although the distractor evoked significant activity before the saccade, it had no effect on any measure of saccadic performance: velocity, reaction time, accuracy, or even the early trajectory. 4

\section{DYNAMICS OF ATTENTION IN THE MONKEY}

Neurons in LIP clearly do not have information about where or when a saccade will occur, although they do respond to saccade targets. It is possible, however, that neurons in LIP specify the locus of attention. If this were true, the activity in the distractor task enables two predictions about attention in the monkey: (1) the default location of attention during the delay period of the delayed saccade task is the saccade goal; and (2) a distractor appearing during a delayed saccade will transiently pull attention away from the saccade goal even though it will not disrupt the saccade plan. It is known in humans that attention is ordinarily pinned at a saccade goal ${ }^{12,13}$ and is also drawn to the locus of an abruptly appearing stimulus, ${ }^{14}$ but the interaction of these effects has not been studied in humans or monkeys.

The locus of attention has been determined in two different ways. It is the area of space associated with the shortest reaction times ${ }^{2}$ or the lowest perceptual threshold. ${ }^{15}$ Because using perceptual thresholds would enable us to study the temporal as well as the spatial aspects of attention, we trained two rhesus monkeys on a perceptual task. ${ }^{16}$ The task began with a monkey fixating on a spot for 1,000-1,200 ms after which a saccade target appeared and disappeared at one of four loci equidistant from the fovea and reflected across the horizontal or vertical meridia. Then 500 to 1,500 ms later a cue appeared for one video frame (roughly $17 \mathrm{~ms}$ ). The cue consisted of a stimulus at each possible saccade goal: three circles and a Landolt ring with its gap on either the right or the left. If the gap were on the left, the monkey had to make the saccade when, $500 \mathrm{~ms}$ later, the fixation point disappeared. If the gap were on the right, the monkey had to continue fixating. Then we were able to measure a contrast sensitivity curve for the cue, which easily fit a classic Weibull function. We defined the threshold contrast sensitivity as that contrast at which the monkey perceived the cue $75 \%$ of the time. When the cue appeared at the saccade goal, the threshold was at $1 \%$ contrast above background. When the cue appeared at any of the other sites (the other possible saccade goals used during that experimental day), the threshold was at $2 \%$ contrast. There were two levels of contrast sensitivity, the advantaged one at the saccade goa, and the unadvantaged level elsewhere. The saccade goal was perceptually advantaged throughout the delay period, even 1,700 $\mathrm{ms}$ after the appearance of the saccade target. Thus, the default locus of attention rests at the goal of a memory-guided saccade task. 
In the next version of the task we inserted a task-irrelevant distractor between the target and the cue to see if the distractor could draw the locus of attention away from the cue. Five hundred milliseconds after the saccade target appeared the distractor, identical to the saccade target, appeared for $100 \mathrm{~ms}$ at one of the four possible cue locations. After a variable period, the cue appeared for one video frame, and the fixation point disappeared $500 \mathrm{~ms}$ later. Two hundred milliseconds after the distractor appeared, the contrast sensitivity at the distractor site was at the advantaged level, and the contrast sensitivity at the saccade goal was at the unadvantaged level. Six hundred milliseconds after the distractor appeared, the saccade goal was again advantaged, as if the locus of attention had moved from the distractor site back to the saccade goal.

Neurons in LIP during this task had activity paralleling the movement of the attentional focus. The saccade target evoked a large burst of activity, which then fell to a delay period level significantly above background, but lower than the peak of the burst. The distractor evoked a large response, which on average returned to a level below the delay period activity between $340 \mathrm{~ms}$ and $455 \mathrm{~ms}$ depending on the monkey. If the cue flashed at the time when the activity evoked by the distractor was identical to the delay period activity for a given monkey, there was no attentional advantage at either site. The delay period activity was clearly greater than the distractor activity for the first monkey at the time curves crossed for the second monkey. There was a clear attentional advantage at the saccade goal for the first monkey at that time.

\section{DISCUSSION}

In these experiments we have demonstrated two different aspects of the representation of the salient visual world in LIP. These different aspects correspond to the objects of James' different kinds of attention. Recently appeared objects evoke James' passive, reflex, or involuntary attention and they also evoke responses in LIP even when they are irrelevant to the ongoing task, whether they appear in the receptive field directly or enter the receptive field by a saccade shortly after their appearance. Sudden motion also evokes activity in LIP, in a directionally nonselective fashion. $^{3}$

Attended stable objects evoke James' active or voluntary attention. Such objects are also represented in LIP. They are not salient by themselves, but become so by virtue of their relevance to the task. Whether they have just entered the receptive field or have been in the receptive field already, they evoke a response much greater than that evoked by stable objects that are irrelevant to the animal's behavior.

Neurons in LIP discharge throughout the interval of a memory-guided delayed saccade task. They respond to the abrupt onset of the stimulus, continue to discharge during the delay period, and burst slightly before the saccade. This pattern of activity has led a number of investigators to assume that LIP is providing a motor intention signal for the saccade. ${ }^{17,18}$ However, there are many examples that demonstrate instances for which the signal in LIP contradicts a saccade plan: LIP neurons describe stimuli that describe saccades elsewhere ${ }^{9}$ and respond in an enhanced manner to stimuli that flash in the receptive field of a neuron when a monkey is planning a saccade elsewhere. The distractor does not interfere with the performance of the saccade, even though immediately before the saccade one cannot distinguish between 
activity evoked by a memory-guided saccade to the receptive field when a distractor appeared elsewhere and a distractor in the receptive field when the saccade goal is elsewhere. Neurons in LIP respond to saccade targets, but discharge much less before saccades not made to visual targets. ${ }^{9}$ This is in distinction to movement neurons in the frontal eye field which are defined by their equivalent discharge in association with visually guided and learned saccades. ${ }^{19}$ Neurons in LIP do not predict where, when, or if a saccade will occur.

Instead, the activity of neurons in LIP describes salient spatial locations, and the area of the visual field associated with the greatest activity in LIP corresponds to the locus of visual attention. This is a winner-take-all mechanism rather than a graded one. The intensity of discharge in LIP does not correlate with the amount of attention; instead, it correlates with the probability that a given location will win. ${ }^{20}$ Ordinarily the locus of visual attention is pinned at the saccade goal throughout the delay period of a memory-guided saccade, and this is consistent with the delay period activity of the parietal neurons as well as with a saccade plan. However, when a distractor appears, the locus of attention moves to the site of the distractor, and this too is consistent with the activity of neurons in LIP, but not with a saccade plan.

\section{ACKNOWLEDGMENTS}

This work was supported by the National Eye Institute and the Human Frontiers Science Program. We are grateful to the staff of the National Eye Institute for assistance in all aspects of this work: Drs. James Raber and Ginger Tansey for veterinary care; Dr. John McClurkin for display programming; Thomas Ruffner and Altah Nichols for machining; Lee Jensen for electronics; Mitchell Smith for histology; Art Hays for computer systems; Brian Keegan for technical assistance; and Becky Harvey and Jean Steinberg for facilitating everything. The Laboratory of Diagnostic Radiology of the Clinical Center provided MRI services.

\section{REFERENCES}

1. James, W. 1890. The Principles of Psychology. Holt. New York.

2. Posner, M.I. 1980. Orienting of attention. Q. J. Exp. Psychol. 32: 3-25.

3. Kusunoki, M., J. GotTlieb \& M.E. Goldberg. 2000. The lateral intraparietal area as a salience map: the representation of abrupt onset, stimulus motion, and task relevance. Vision Res. 40: 1459-1468.

4. Powell, K.D. \& M.E. Goldberg. 2000. Response of neurons in the lateral intraparietal area to a distractor flashed during the delay period of a memory-guided saccade. J. Neurophysiol. 84: 301-310.

5. SPARKS, D.L. 1975. Response properties of eye movement-related neurons in the monkey superior colliculus. Brain Res. 90: 147-152.

6. HikosaKa, O. \& R.H. Wurtz. 1983. Visual and oculomotor functions of monkey substantia nigra pars reticulata. III. Memory-contingent visual and saccade responses. J. Neurophysiol. 49: 1268-1284.

7. WURTZ, R.H. 1969. Visual receptive fields of striate cortex neurons in awake monkeys. J. Neurophysiol. 32: 727-742.

8. Egeth, H.E. \& S. Yantis. 1997. Visual attention: control, representation, and time course. Annu. Rev. Psychol. 48: 269-297. 
9. Gottlieb, J., M. Kusunoki \& M.E. Goldberg. 1998. The representation of visual salience in monkey parietal cortex. Nature 391: 481-484.

10. Duhamel, J.-R., C.L. Colby \& M.E. Goldberg. 1992. The updating of the representation of visual space in parietal cortex by intended eye movements. Science 255: 90 92.

11. Snyder, L.H., A.P. Batista \& R.A. Andersen. 1997. Coding of intention in the posterior parietal cortex. Nature VI-386: 167-170.

12. Deubel, H. \& W.X. SchneIDER. 1996. Saccade target selection and object recognition: evidence for a common attentional mechanism. Vision Res. 36: 1827-1837.

13. Kowler, E., E. Anderson, B. Dosher \& E. Blaser. 1995. The role of attention in the programming of saccades. Vision Res. 35: 1897-1916.

14. YANTIS, S. \& J. JONIDES. 1984. Abrupt visual onsets and selective attention: evidence from visual search. J. Exp. Psychol. Hum. Percept. Perform. 10: 601-621.

15. BASHINSKI, H.S. \& V.R. BACHARACH. 1980. Enhancement of perceptual sensitivity as the result of selectively attending to spatial locations. Percept. \& Psychophys. 28: 241-248.

16. Bisley, J.W. \& M.E. Goldberg. 2001. The locus of visual attention can be predicted by the responses of neurons in the lateral intraparietal area. Soc. Neurosci. Abstr. 27: 384.

17. Andersen, R.A., L.H. Snyder, D.C. Bradley \& J. XING. 1997. Multimodal representation of space in the posterior parietal cortex and its use in planning movements. Annu. Rev. Neurosci. 20: 303-330.

18. Platt, M.L. \& P.W. Glimcher. 1997. Responses of intraparietal neurons to saccadic targets and visual distractors. J. Neurophysiol. 78: 1574-1589.

19. BRUCE, C.J. \& M.E. GoldBerg. 1985. Primate frontal eye fields: I. Single neurons discharging before saccades. J. Neurophysiol. 53: 603-635.

20. Desimone, R. \& J. Duncan. 1995. Neural mechanisms of selective visual attention. Annu. Rev. Neurosci. 18: 183-222. 